\title{
Reconstitution of male germline cell specification from mouse embryonic stem cells using defined factors in vitro
}

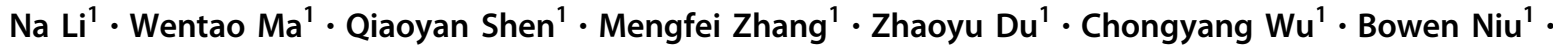 \\ Wenqing Liu ${ }^{1} \cdot$ Jinlian Hua ${ }^{1}$
}

Received: 17 June 2018 / Revised: 24 November 2018 / Accepted: 2 January 2019 / Published online: 25 January 2019

(c) ADMC Associazione Differenziamento e Morte Cellulare 2019

\begin{abstract}
In vitro induction of functional haploid cells from embryonic stem cells (ESCs) has been reported by several groups. However, these reports either involve complex induction process with undefined induction factors or show low-induction efficiency. Here, we report complete meiosis in vitro from ESCs with defined induction factors. ESCs were first induced into primordial germ cell-like cells, which were further induced into male germline cells, including spermatogonial stem cell-like cells (SSCLCs) and spermatid-like cells. Importantly, the obtained SSCLCs were functional as infertile male mice sired healthy offspring via SSCLC transplantation. Further, we found that eukaryotic translation initiation factor 2 subunit 3 and structural gene Y-linked (Eif2s3y) was essential for spermatogenesis. Eif2s3y-overexpressing ESCs showed enhanced spermatogenesis in vitro, as demonstrated by higher expression levels of SSC-specific markers during SSCLC induction process, improved reproductive ability recovery of infertile male mice, and increased efficiency of haploid cell induction. Our work provides a convenient and efficient approach to obtain functional male germline cells.
\end{abstract}

\section{Introduction}

Infertility is a widespread global issue. In approximately one in five infertile couples, the problem lies solely in the male partner, with one of the main factors being a lack of sperms in the testis. Spermatogonial stem cell (SSC) induction and transplantation is promising to tackle this problem. However, germ cell development is a complex multistage biological process, making it difficult for reproducing germ cell development in vitro.

In 2011, Hayashi et al. [1] reported the reconstitution of mouse primordial germ cell-like cell (PGCLC) specification pathway from pluripotent stem cells (PSCs). Later, two

\section{Edited by M. Piacentini}

Supplementary information The online version of this article (https:// doi.org/10.1038/s41418-019-0280-2) contains supplementary material, which is available to authorized users.

Jinlian Hua

jinlianhua@nwsuaf.edu.cn

1 College of Veterinary Medicine, Shaanxi Center of Stem Cells Engineering \& Technology, Northwest A \& F University, Yangling, Shaanxi, China independent groups reported the establishment of functional male gamete induction system from PSCs in vitro [2, 3]. However, these two reports involve coculture of PGCLCs with neonatal or embryonic testicular somatic cells, making the culture process more complicated and difficult to determine what factor(s) secreted by the testicular somatic cells are responsible for spermatogenesis. In 2018, Zhao et al. [4] established a feeder- and xeno-free culture protocol that induced human PSCs into spermatogonium-like cells. However, their induction efficiency was rather low (4.9\%).

Eukaryotic translation initiation factor 2 subunit 3 and structural gene Y-linked (Eif2s3y) is essential for mouse spermatogenesis [5-8]. Upon the transgenic addition of sexdetermining region Y (SRY) and Eif2s3y to male mice with an $\mathrm{X}$ chromosome but without a $\mathrm{Y}$ chromosome, a substantial number of spermatocytes complete the first meiotic division [9], with the occasional production of spermatidlike cells (SLCs) [5, 9]. In our previous study, we found that embryonic stem cell (ESC)-derived cell lines overexpressing Eif2s3y showed reduced pluripotency and faster proliferation rate [5]. Thus, it is tempting to verify whether Eif2s $3 y$ overexpression improves the efficiency of spermatogenesis in vitro.

Here, we used an in vitro culture protocol with defined induction factors to reconstitute the development of male 

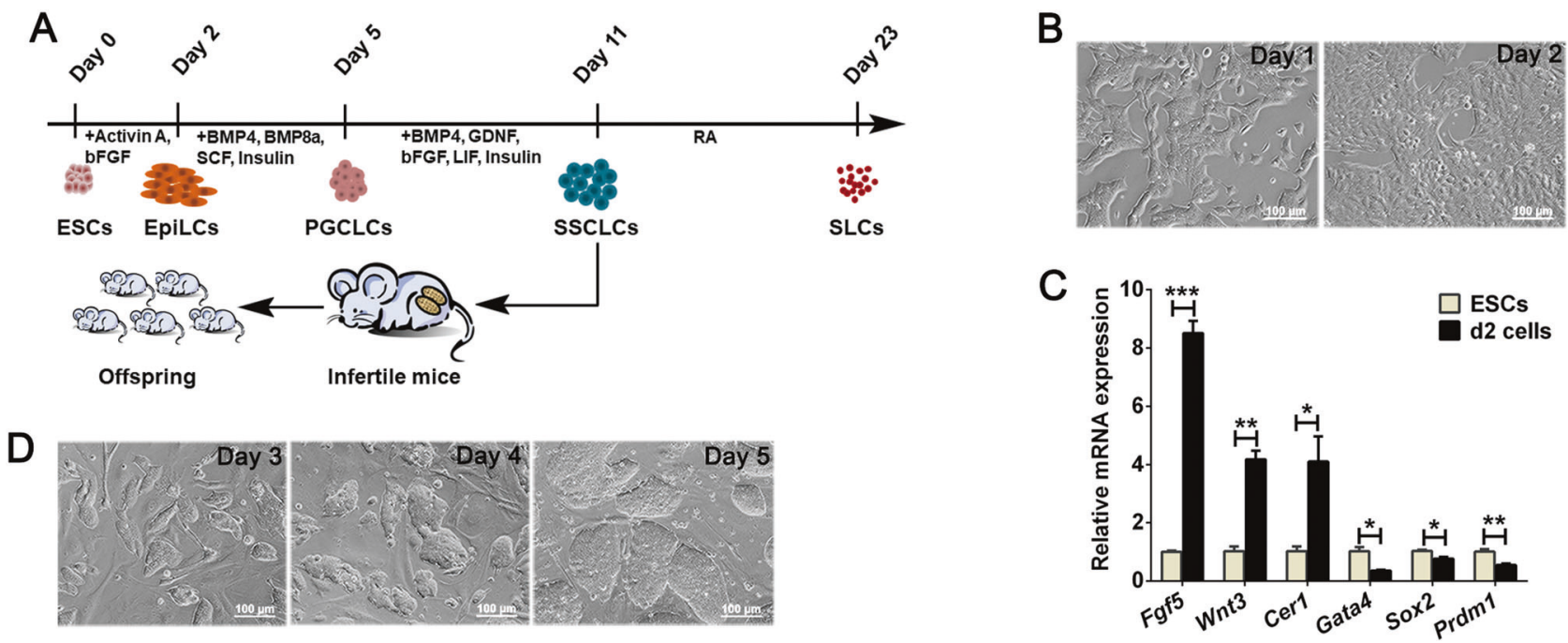

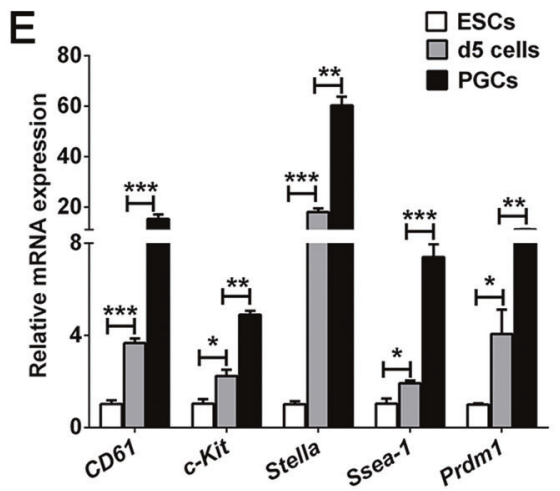

Fig. 1 Generation of EpiLCs and PGCLCs from ESCs. a The male germline cell induction system and timeline. b Bright-field images of the day 1- and day 2-induced cells. Scale bar, $100 \mu \mathrm{m}$. c Real-time PCR analysis of Fgf5, Wnt3, Cerl, Gata4, Sox2, and Prdm1 expression levels in ESCs and the day 2-induced cells. d Bright-field images of the induced cells during day 3 to day 5 . Scale bar, $100 \mu \mathrm{m}$. e Realtime PCR analysis of CD61, c-Kit, Stella, Ssea-1, and Prdm1 in ESCs,

germline cells from ESCs. The obtained male germline cells were functional, as transplantation of the induced SSCLCs restored the reproductive ability of male infertile mice. In addition, we found that Eif2s $3 y$ overexpression promoted meiosis in vitro. Our study provides an efficient approach to reconstitute the entire process of meiosis in culture and a potential cellular therapy for male infertility.

\section{Results}

\section{Generation of EpiLCs and PGCLCs from ESCs}

The ESCs used in this system was labeled by an acrosinDsRed promoter (Fig. S1), the primers are listed in Table S1. Epiblast-like cells (EpiLCs) and PGCLCs were induced from ESCs adapted from a previous report [1]. Upon the stimulation with Activin A, basic fibroblast growth factor (bFGF), the day 5-induced cells and E12.5 PGCs. f Immunofluorescence staining of SSEA-1, PRDM1, PRDM14, and AP2 $\gamma$ in day 5-induced cells, counterstained with Hoechst 33342. Scale bar, $50 \mu \mathrm{m}$. g Flow cytometric analysis of CD61 and SSEA-1 expression of the day 5induced cells. Data are represented as mean \pm SEM from more than three independent experiments, $* p<0.05$, $* * p<0.01, * * * p<0.001$

and knockout serum replacement (KSR) (Fig. 1a), the cell clones became flat and grew rapidly over 2 days (Fig. 1b). The expression of epiblast-specific genes, such as Fgf5, Wnt3, and Cerl were confirmed by real-time polymerase chain reaction (PCR) analysis, which showed that these genes significantly upregulated in day 2 -induced cells (Fig. 1c). The expression levels of the primitive endoderm marker Gata4 and pluripotency markers $\operatorname{Sox} 2$ and $\operatorname{Prdm} 1$ were significantly downregulated in day 2-induced cells (Fig. 1c). Next, the day 2-induced cells were reseeded on a 12-well plate containing a feeder layer, and the cultured medium supplemented with bone morphogenetic protein 4 (BMP4), BMP8a, stem cell factor (SCF), insulin, and KSR. From day 3 to day 5, the cells exhibited a feature of clones with smooth edges, and the clones became larger during culture (Fig. 1d). As reported previously [1], we chose the day 5 -induced cells for further analysis. Our results demonstrated that these cells upregulated PGC-specific genes such as CD61, c-Kit, Stella, Ssea- 
A
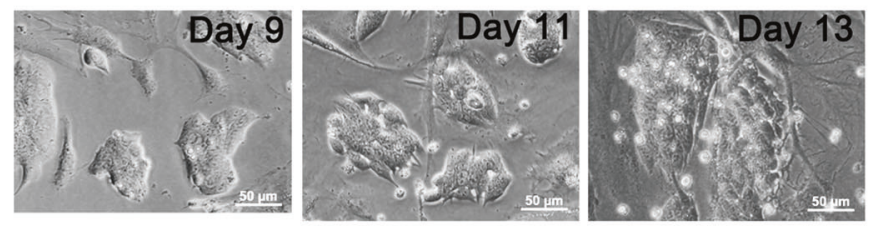

C

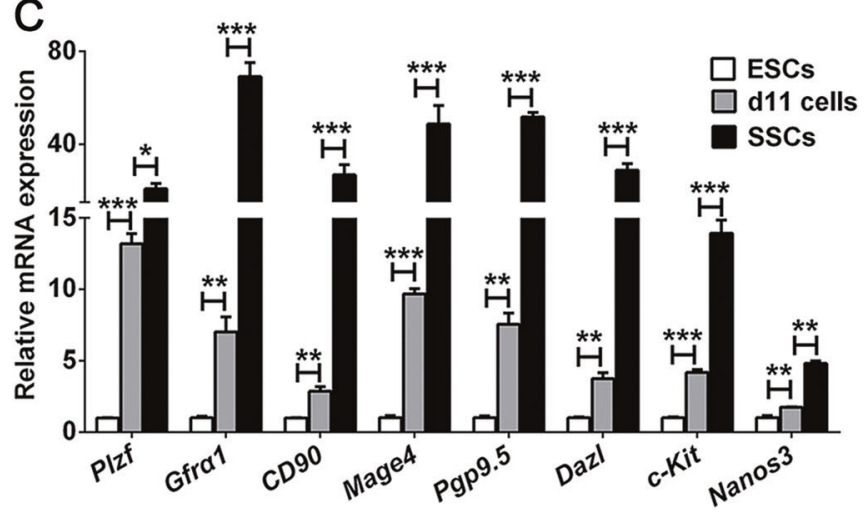

E

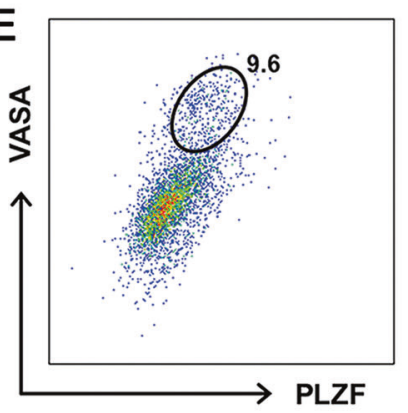

Fig. 2 Identification of the characteristic markers for SSCLCs. a Bright-field images of the induced cells at day 9, day 11, and day 13 . Scale bar, $50 \mu \mathrm{m}$. b Gene expression dynamics of Plzf, Gfral, CD90, Dazl, and $c$-Kit during the SSCLC induction at day 9, day 11, and day 13. c Real-time PCR analyses of Plzf, Gfra1, CD90, Mage4, Pgp9.5, Dazl, c-Kit, and Nanos3 of ESCs, day 11-induced cells, and SSCs from postnatal day 7 mice. d Immunofluorescence analysis of PLZF,

1, and Prdml significantly (Fig. 1e). Moreover, immunofluorescence staining showed that the day 5-induced cell clusters expressed PGC-specific markers, i.e., SSEA-1, PRDM1, PRDM14, and AP2 $\gamma$ (Fig. 1f). To determine the percent of PGCLCs in the day 5-induced cells, we further used flow cytometry to analyze the expression profile of SSEA1 and CD61, the co-expression of which could serve as a PGC-specific marker. The result turned out that SSEA1 and CD61 double-positive cells was 14\% (Fig. 1g).

\section{In vitro differentiation of PGCLCs into SSCLCs}

According to previous reports, either transplantation of PGCLCs directly into infertile male mice or coculture with testicular cells and humoral stimulation could induce the
B

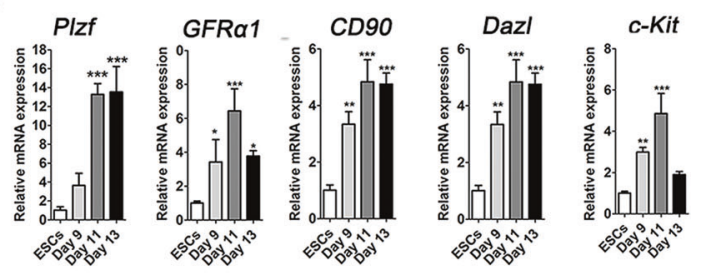

D
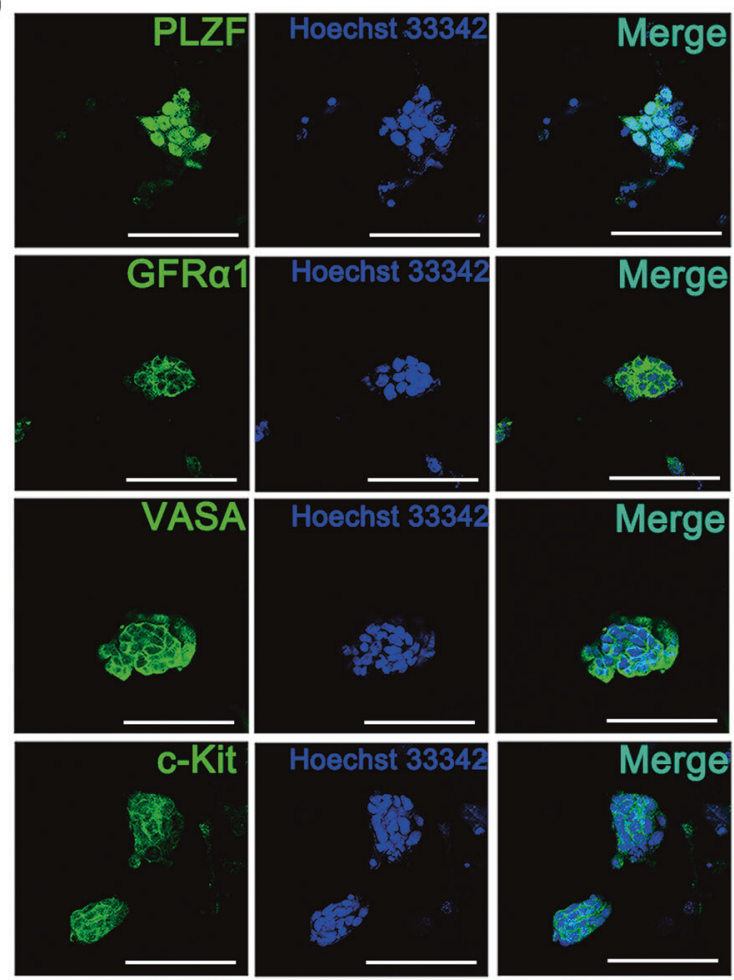

GFR $\alpha 1$, VASA, and c-Kit of the day 11-induced cells, counterstained with Hoechst 33342. Images are representative isolated clusters positive for the indicated SSC-specific markers. Scale bar, $50 \mu \mathrm{m}$. e Flow cytometric analysis of VASA and PLZF expression of the day 11induced cells. Data are represented as mean \pm SEM from more than three independent experiments, $* p<0.05, * * p<0.01, * * * p<0.001$.

meiosis process $[1,3]$. We wondered whether this could be achieved with specific cytokines in vitro. We reseeded day 5-induced cells onto feeder cells and supplemented the culture with BMP4, glial cell-derived neurotrophic factor (GDNF), bFGF, leukemia inhibitory factor (LIF), insulin and KSR in the culture medium (Fig. 1a). During the culture process, most of the cell clones were closely arranged, and with unclear borders. From day 11, several compact clusters with clear cell borders appeared (Fig. 2a). Gene-expression analysis revealed that spermatogonial stem cell markers Plzf, Gfral, CD90, Dazl, and c-Kit all increased in transcript levels from day 9 and peaked at day 11 (Fig. 2b). Based on this, we chose the day 11-induced cells for subsequent experiments. Using real-time PCR analysis, we found that the expression of SSC self-renewal markers, such 
A
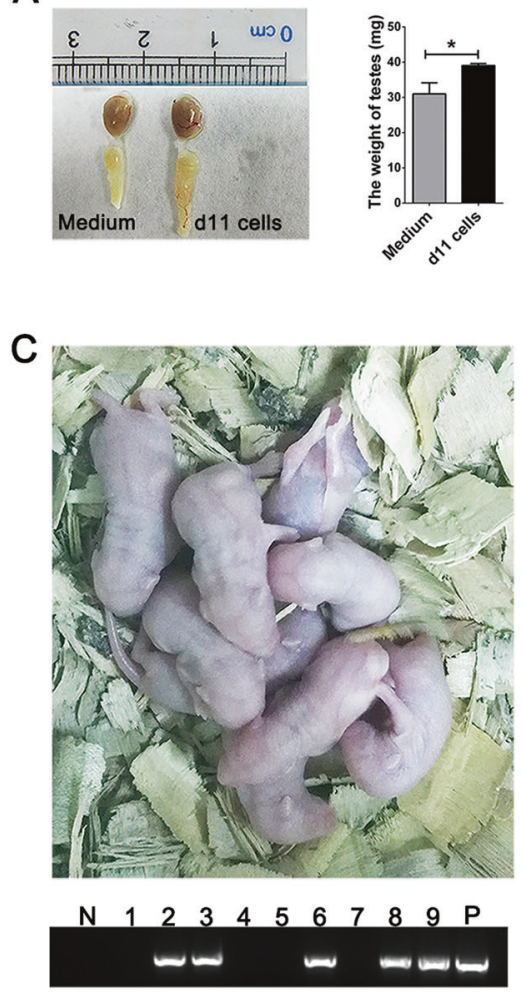

B

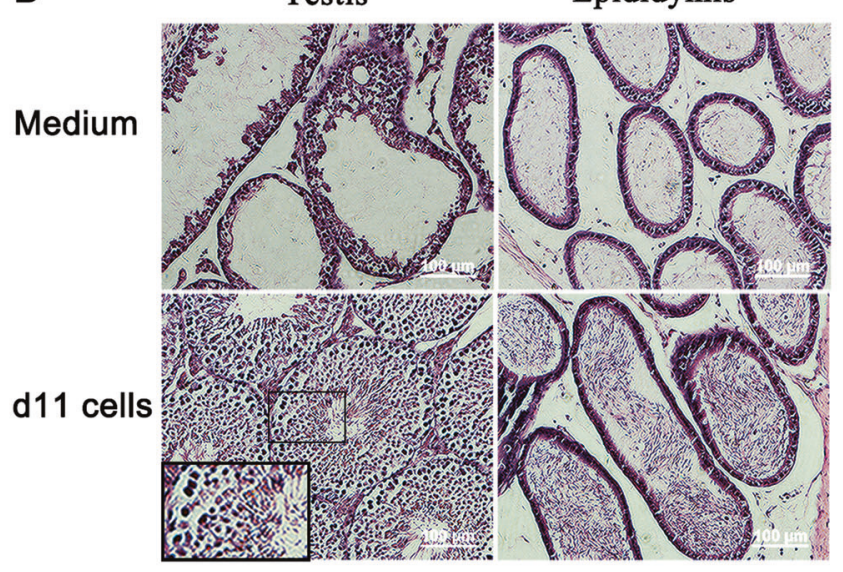

D

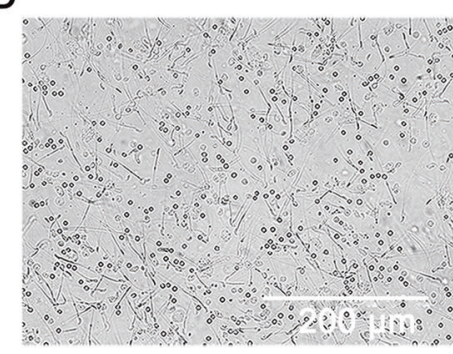

Bright-field

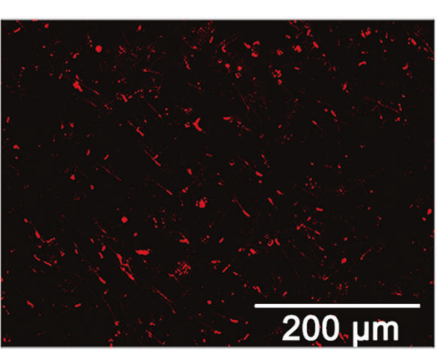

Acrosin-DsRed
Fig. 3 Recovery of reproductive ability of infertile male mice via day 11 -induced cell transplantation. a Image of testes subjected to seminiferous tubule transplantation of medium alone and day 11-induced cells in medium with the same volume (Day 11) after 2 months of transplantation (left panel). The weight of these testes is quantified in the bar chart (right panel). b Representative HE staining of medium- or day 11-induced cell-transplanted testes and epididymis. Box indicates the seminiferous tubules at stage VIII-IX of the seminiferous

as Plzf, Gfral, CD90, Mage4, and Pgp9.5, and differentiating markers, including Dazl, c-Kit, and Nanos3, were significantly increased in these day 11-induced cells (Fig. 2c). Moreover, immunofluorescent staining analysis showed that the representative clusters were positive for PLZF, GFR $\alpha 1$, VASA, and c-Kit (Fig. 2d). The doublepositive cells for VASA and PLZF was $9.6 \%$ as quantified by flow cytometry (Fig. 2e). These findings indicate that the induced PGCLCs were responsive to BMP4, GDNF, bFGF, LIF and insulin, which are critical for male germ cell fate decision.

To confirm whether the induced-cells can restore fertility of infertile male mice, we transplanted these cells into the seminiferous tubules of busulfan-treated azoospermia male mice [10-12]. As expected, transplantation of day 11-induced cells restored fertility of recipient mice. Mice with day 11induced cell transplantation showed significantly greater testis weight compared with those transplanted with medium only (Fig. 3a). In addition, hematoxylin and eosin (HE) staining revealed that all types of male germ cells could be observed in the day 11-induced cell-transplanted testes and the epididymis epithelium cycle. Scale bar, $100 \mu \mathrm{m}$. c Live offspring of day 11induced cell-transplanted male infertile mice. Genotyping for acrosinDsRed-transgene of the pups is shown at the bottom. d Observation of semen smears from the 2-month-old offspring in (c). Left, bright-field image. Right, expression profile of acrosin-DsRed observed through a fluorescent microscope. Scale bar, $200 \mu \mathrm{m}$. Data are represented as mean $\pm \mathrm{SEM}$ from more than three independent experiments, ${ }^{*} p<$ 0.05 .

was filled with mature spermatozoa, while mediumtransplanted testes had few cells alongside the basement membrane, and few mature spermatozoa were observed in the epididymis (Fig. 3b). Instead, ESC transplantation formed teratomas in the recipient testes as shown in Fig. S2. To confirm whether the day 11-induced cell transplantation could restore reproductive capacity of infertile mice, the recipient mice were mated with wild-type females after 8 weeks of transplantation. The results showed that the day 11-induced cell-transplanted recipients sired 9 live offspring (Fig. 3c). At the age of 2 months, semen smears of the epididymis of the male offspring were made. As can be seen from Fig. 3d, large numbers of acrosin-positive mature sperms were observed.

\section{Induction of haploid cells from SSCLCs in vitro}

Meiosis is the key step of gametogenesis, in mammals, retinoic acid (RA) is critical for meiosis initiation [13]. In our study, we further cultured the day 11-induced cells in basal culture medium supplemented with RA. During the culture process, small round cells that were acrosin-positive 

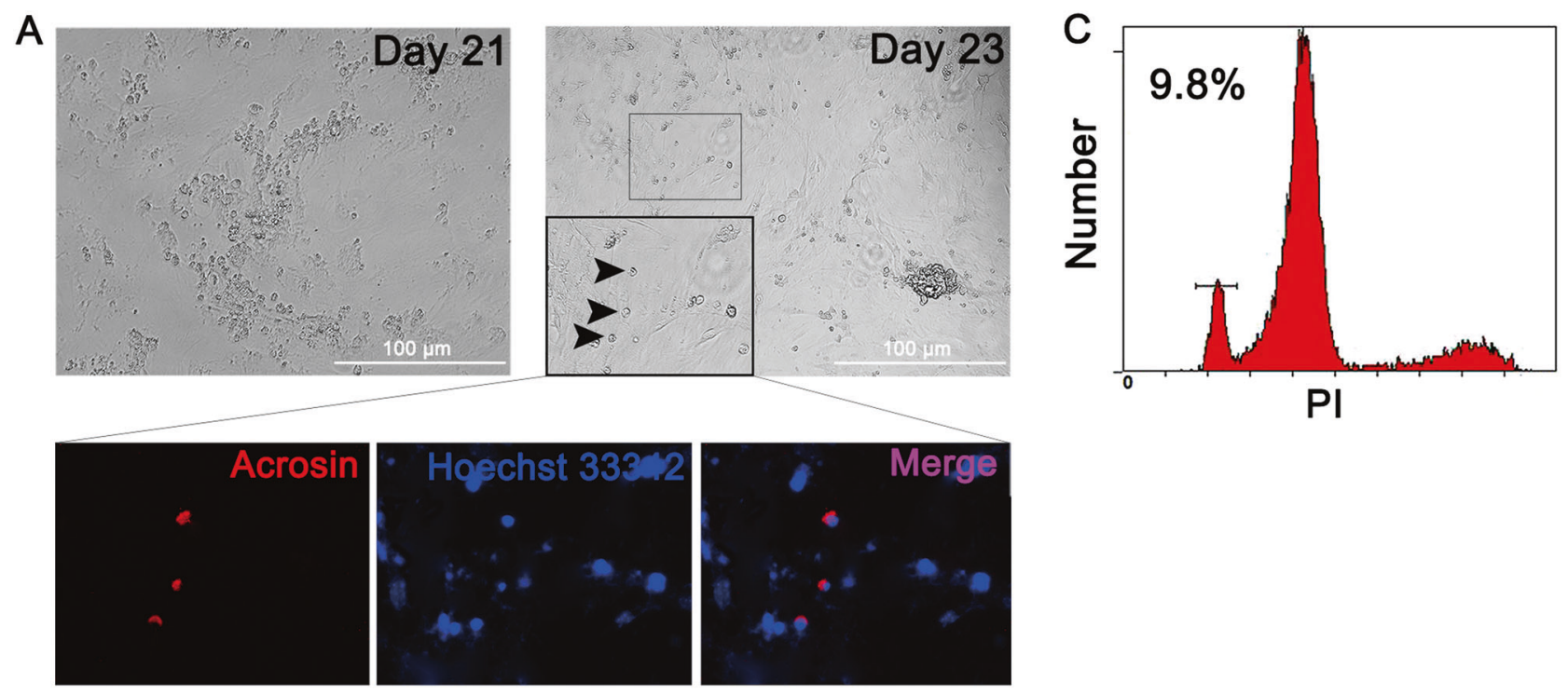

B
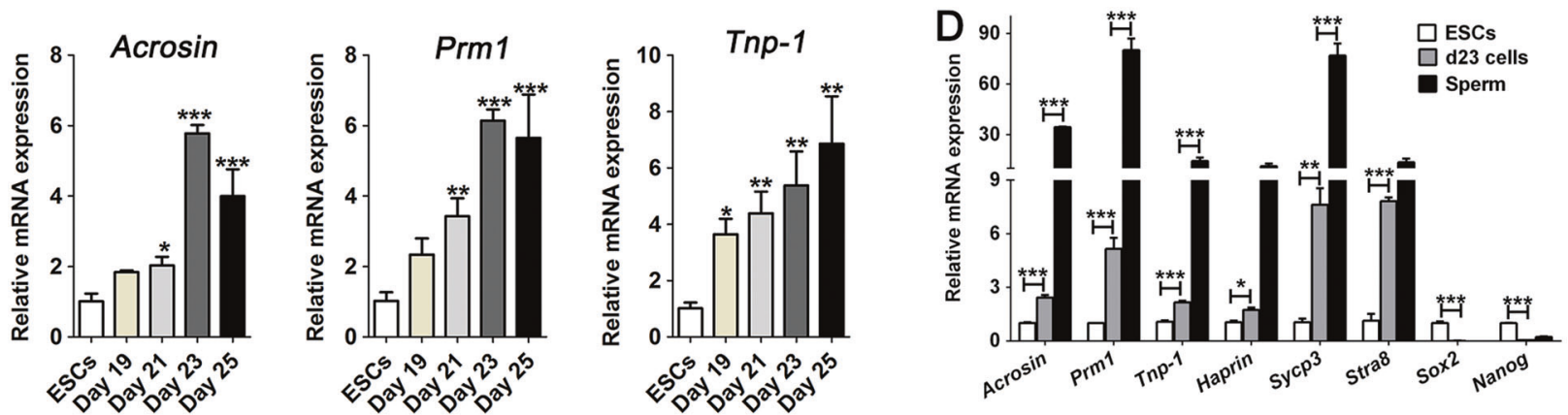

Fig. 4 Haploid SLCs appeared in the male germline cell induction system in vitro. a Images of the day 21 - and day 23-induced cells. Upper panel, bright-field images of the day 21- and day 23-induced cells, arrowheads indicate small round haploid cells. Lower panel, acrosin-DsRed expression profile of these small round cells at day 23 . Scale bar, $100 \mu \mathrm{m}$. b Real-time PCR analyses of acrosin, Prm1 and Tnp-1 expression levels during the induction process at day 19, day 21 ,

appeared (Fig. 4a). Dynamic analysis of gene expression revealed that spermatid markers acrosin, Prm1 and Tnp-1 all increased and peaked at day 23 (Fig. 4b). Flow cytometry analysis showed that the percentage of haploid cells in the day 23 -induced cells was $9.8 \%$ (Fig. 4c). In addition, the transcripts of the day 23-induced cells have significantly changed. Among these transcripts, spermatid-specific markers Acrosin, Prml, Tnp-1, and Haprin, along with meiosisassociated markers Sycp3 and Stra8, were significantly increased, while pluripotency-associated markers Sox 2 and Nanog were reduced as expected [14] (Fig. 4d). Therefore, our induction system reconstituted complete meiosis and generated SLCs from ESCs in vitro.

\section{Eif2s3y improves the efficiency of SSCLC induction}

Eif2s $3 y$ is essential for spermatogenesis and can rescue early spermatogenesis failure in $\mathrm{Y}$ short arm-deleted mice [15]. day 23, and day 25. c Flow cytometric analysis of the percentage of haploid cells of day 23-induced cells. d Real-time PCR analyses of acrosin, Prm1, Tnp-1, Haprin, Sycp3, Stra8, Sox2, and Nanog expression level of ESCs, day 23-induced cells and sperms. Data are represented as mean \pm SEM from more than three independent experiments, $* p<0.05$, ** $p<0.01$, *** $p<0.001$.

Additionally, offspring could be produced using germ cells from males with $\mathrm{Y}$ chromosome contributions that were limited to only two genes (Sry and Eif2s3y) [9]. Here, we introduced Eif2s3y in meiosis reconstitution using ESCs in vitro. In this experiment, ESCs overexpressing Eif2s3y or the vector pTRIPCAGG-pur were used, as we previously reported [5].

Interestingly, Eif2s3y-overexpressing ESCs showed significantly higher ratio of SSEA-1 and CD61 double-positive cells at day 5-induced cells (13.7\% vs. $22.8 \%$ ) (Fig. S3), and at day 11, the percentage of VASA and PLZF double-positive cells was significantly higher in Eif2s3y group relative to the Vector group (9.4\% vs. $37.3 \%$ ) (Fig. 5a, b). Further RNAsequence analysis for 11-induced cells, comparing genes associated with pluripotency, self-renewal SSCs, differentiating SSCs, endoderm, and ectoderm showed that the expression pattern of the Eif2s3y group were closer to that of testis (Fig. 5c). Then, the day 11-induced cells from the Vector-and Eif2s3y-overexpressing ESCs were transplanted into the 
A

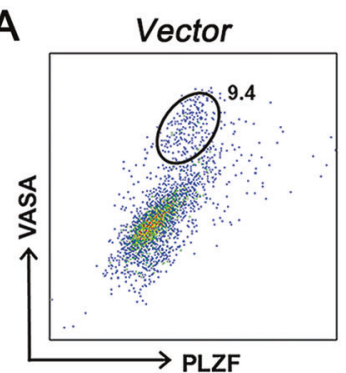

D

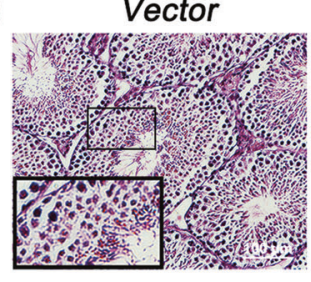

F

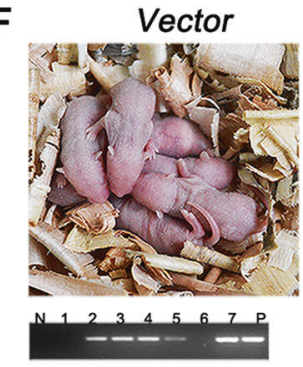

Eif2s3y

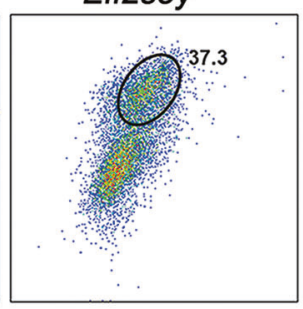

Eif2s3y

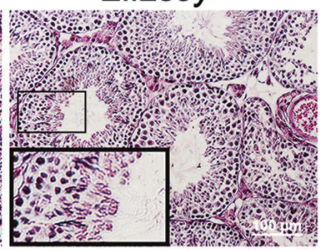

Eif2s3y

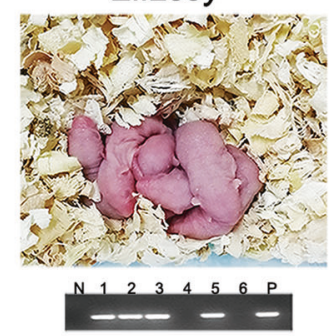

G

\begin{tabular}{|l|c|c|}
\hline & $\begin{array}{c}\text { Number of } \\
\text { recipient infertile mice }\end{array}$ & $\begin{array}{c}\text { Number of reproductive } \\
\text { ability-restored mice }\end{array}$ \\
\hline Vector & 7 & 1 \\
\hline Eif2s3y & 7 & 2 \\
\hline
\end{tabular}

$\mathrm{H}$

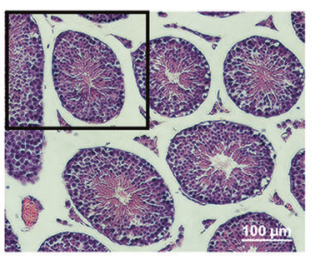

B

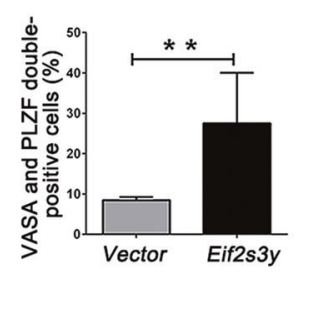

E

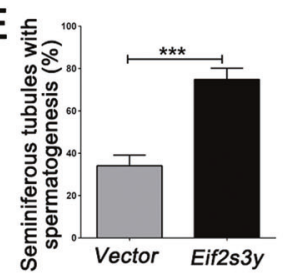

I

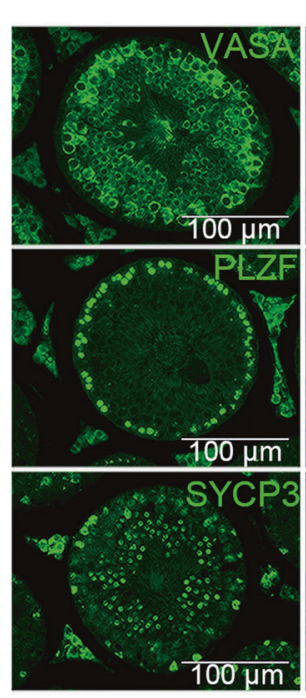

C
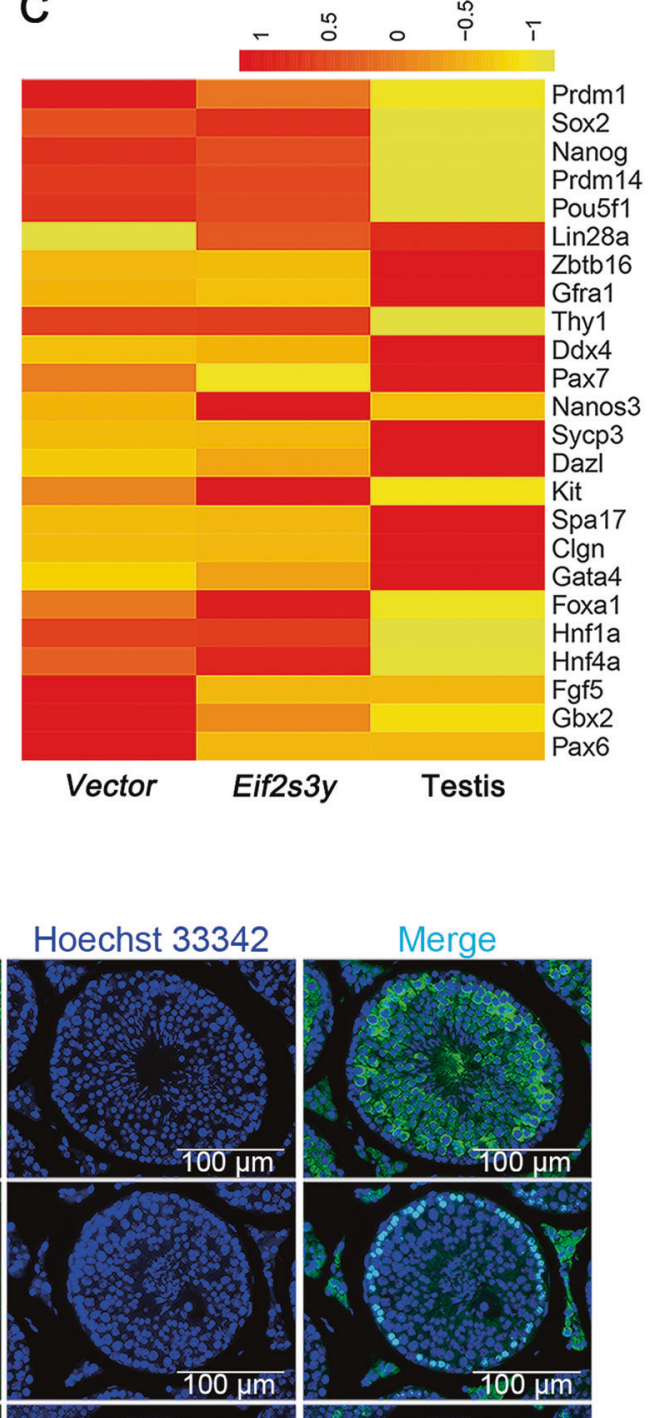

$100 \mathrm{\mu m}$
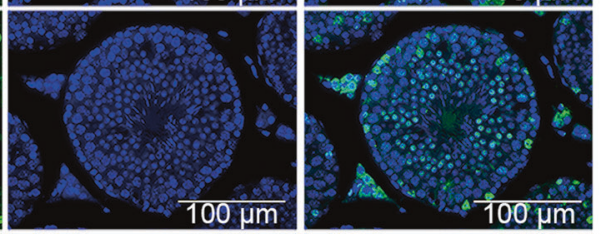

Fig. 5 Comparisons of SSCLCs induced from ESCs overexpressing empty vector or Eif2s3y. a, b Representative flow cytometric analysis (a) and quantification (b) of VASA and PLZF double-positive cell percent of day 11-induced cells that are either from ESCs overexpressing empty vector (Vector) or overexpressing Eif2s3y (Eif2s3y). c Heat map of representative markers of pluripotency, self-renewing SSCs, differentiating SSCs, endoderm and ectoderm in the day 11induced cells derived from ESCs overexpressing empty vector (Vector), ESCs overexpressing Eif2s3y (Eif2s3y). Testis from a wild-type 2-month-old mouse was used as a positive control for SSCLC. d Representative HE staining of testes of infertile mice that have been transplanted with the day 11-induced cells from ESCs overexpressing empty vector (Vector) or overexpressing Eif2s3y (Eif2s3y) for 8 weeks. Boxes indicate the seminiferous tubules at stage VIII-IX of the seminiferous epithelium cycle. Scale bar, $100 \mu \mathrm{m}$. e The percentage of seminiferous tubules showing spermatogenesis process transplanted with the day 11-induced cells from ESCs overexpressing empty vector (Vector) or overexpressing Eif2s3y (Eif2s3y) for 8 weeks. f Live offspring of male infertile mice obtained by transplantation of the day 11induced cells from ESCs overexpressing empty vector (Vector) or overexpressing Eif2s3y (Eif2s3y). Genotyping of the pups is shown at the bottom. g Reproductive ability recovery profile of infertile male mice via transplantation with the day 11-induced cells from ESCs overexpressing empty vector (Vector) or overexpressing Eif2s3y (Eif2s3y). h Representative HE staining of the testis of a 35-day-old offspring of infertile male mice via transplantation with the day 11induced cells from ESCs overexpressing Eif2s3y. Scale bar, $100 \mu \mathrm{m}$ i Representative immunofluorescence analyses of VASA, PLZF and SYCP3 using samples as indicated in (h). Scale bar, $100 \mu \mathrm{m}$. Data are represented as mean \pm SEM from more than three independent experiments, $* * p<0.01, * * * p<0.001$. 
A

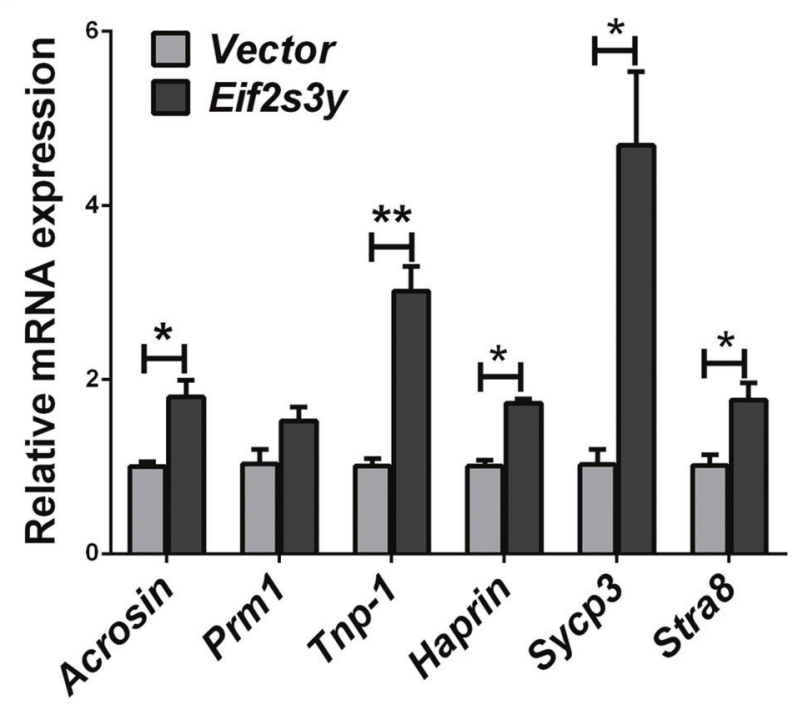

B
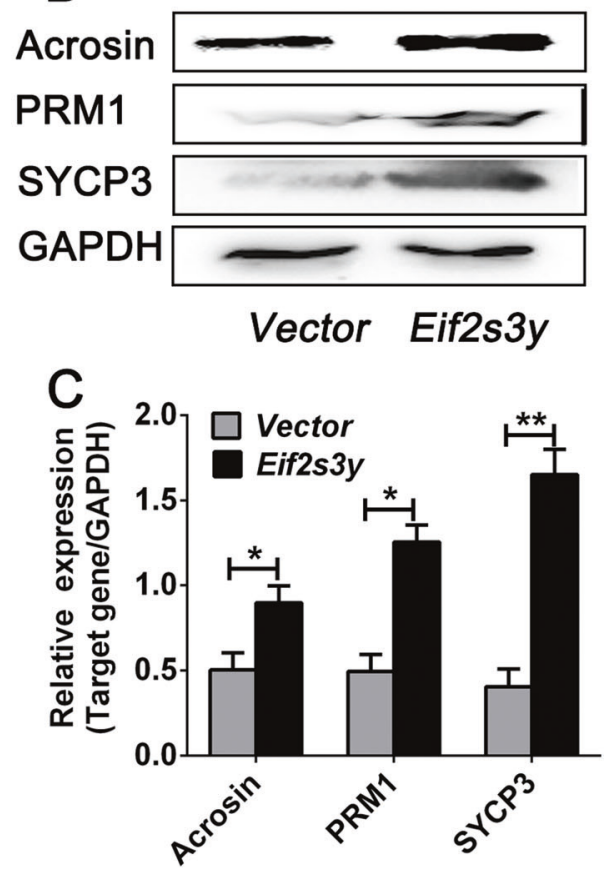

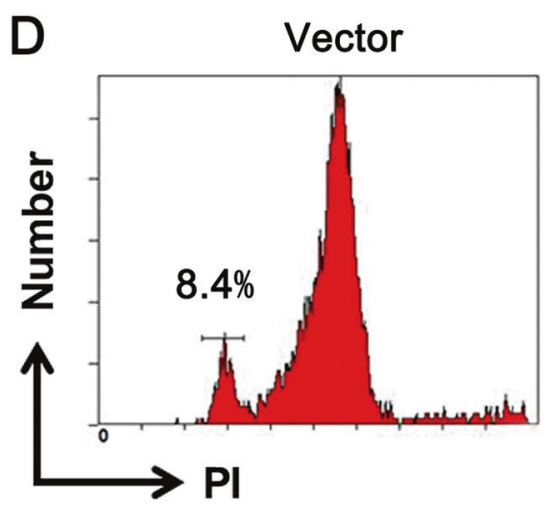

Fig. 6 Comparisons of SLCs induced from ESCs overexpressing empty vector or Eif2s3y. a Real-time PCR analyses of acrosin, Prm1, Tnp-1, Haprin, Sycp3 and Stra8 in day 23-induced cells from ESCs overexpressing empty vector (Vector) or overexpressing Eif2s3y (Eif2s3y). b, c Western blot analyses (b) and quantification (c) of Acrosin, PRM1 and SYCP3 of day 23-induced cells from ESCs

seminiferous tubules of busulfan-treated male mice, respectively. The results showed that Eif2s3y group cell transplantation exhibited more efficient spermatogenesis in vivo, as percentage of seminiferous tubules showing spermatogenesis was significantly higher in this group (75\% vs. $34 \%$, Fig. $5 d$, e). The restored spermatogenesis was functional, as live offspring could be produced in both groups (Fig. 5f). Importantly, Eif $2 s 3 y$ group transplantation resulted in higher numbers of recipient mice which recovered reproductive function (Fig. $5 \mathrm{~g}$ ). The male offspring with Eif2s3y group cell transplantation are healthy, as these mice showed normal seminiferous tubule structure (Fig. 5h) and their testes expressed male germ cell-specific markers, i.e., VASA, overexpressing empty vector (Vector) or overexpressing Eif2s3y (Eif $2 s 3 y)$. d, e Representative flow cytometry results (d) and quantification (e) of the percentage of haploid cells among the day 23induced cells from ESCs overexpressing empty vector (Vector) or overexpressing Eif2s3y (Eif2s3y). Data are represented as mean \pm SEM from more than three independent experiments, ${ }^{*} p<0.05,{ }^{*} p<0.01$.

PLZF, and SYCP3 (Fig. 5i). In addition, the male offspring of Eif2s3y group cell-transplanted mice were fertile and able to have pups (data not shown).

\section{Eif2s3y promotes meiosis in vitro}

We questioned whether Eif2s3y was also important during the subsequent events of spermatogenesis. Accordingly, ESCs overexpressing Eif2s3y or the vector pTRIP-CAGGpur were further subjected to SLC induction. Real-time PCR analysis showed that the day 23-induced Eif $2 s 3 y$ overexpressing ESCs had significantly higher expression levels of Acrosin, Prm1, Tnp-1, Haprin, Sycp3, and Stra8 
(Fig. 6a). Western blot analysis showed a similar result, as Acrosin, PRM1, and SYCP3 were significantly upregulated in protein level in Eif2s3y group (Fig. 6b, c). In addition, DNA content analysis revealed significantly higher percentage of haploid cells in Eif2s3y group (Fig. 6d, e).

Taken together, we generated an efficient approach using all defined factors combined with Eif $2 s 3 y$ overexpression to reconstitute the entire process of meiosis in culture, thus providing help for study of spermatogenesis and a potential therapy for infertility.

\section{Discussion}

Spermatogenesis is a crucial process allowing for the generation of mature male gametes [8]. During its final phase, spermiogenesis, haploid round spermatids differentiates into spermatozoa, which involves extensive and precise restructuring of cell morphology, DNA, and epigenome [8]. It is difficult to elucidate the mechanisms of human spermatogenesis because of the long time period and in vivo development process. Alternatively, various mouse models and in vitro cell culture systems have been used to explore this mechanism. Indeed, the derivation of haploid male gametes could be achieved under the support of embryoid bodies in vitro $[16,17]$. Later, ESCs and iPSCs were induced to generate PGCLCs through EpiLCs in vitro with specific stimulation factors [1]. Recently, functional murine SLCs have been generated in vitro in a specific culture condition in the presence of testicular somatic cells [3]. In addition, the induction of postmeiotic cells or PGCLCs was also achieved using human ESCs and iPSCs [18, 19]. The combination of transcription factors PRDM1, PRDM14, and TFAP2C also induce a PGC fate $[20,21]$. Moreover, murine seminiferous tubules were reconstructed through a $3 \mathrm{D}$ culture system, in which spermatogenesis was progressed to the meiotic phase [22]. Nevertheless, none of these studies reported further induction of SSCLCs and SLCs from PGCLCs with defined stimulation cocktail, and the functionality of the induced cells was not fully evaluated; or even this was tested, the specific induction factors that were responsible for this were obscure. Here, we present a defined combination of in vitro priming factors adapted from previous studies for the successful differentiation of PGCLCs to SSCLCs and further to SLCs $[2,3]$. Moreover, the induced SSCLCs were functional in spermatogenesis and in making female mice pregnant. In comparison with previous works, we have provided detailed proofs for successful meiosis at all induction stages, and for the first time boosted the induction of PGCLCs in vitro, the result of which was proved effective with the production of healthy offspring mice.

In culture conditions in vitro, it is difficult for the inducedPGCLCs to differentiate into SSCLCs, which was likely due to a lack of testicular microenvironment required for SSC differentiation and maintenance, including the growth factors essential for SSC self-renewal. GNDF plays a central role in SSC self-renewal. It was reported that with the presence of only GNDF, or GNDF combined with bFGF, SSCs proliferated robustly and reconstituted long-term spermatogenesis after transplantation into the testes of recipient mice [23]. Besides, the combination of GNDF, bFGF, LIF, and EGF were effective in maintaining long-term SSC culture in vitro, also highlighting the critical roles of these factors for SSC self-renewal [12]. Previous studies have also demonstrated that RA and BMP4 act to promote the differentiation of ESCs into germ cells, and even functional gametes, which can initiate and support in vivo development after intracytoplasmic injection [3, 24, 25]. The entrance into meiosis is stimulated by RA and mediated by the Stra8 gene [24, 26]. Based on these studies, we have screened several stimulation systems for the induction of PGCLCs to SSCLCs and further to SLCs, and finally optimized the most efficient combination for this stimulation, as reported here. In line with previous studies [2, 4, 12, 26], we also highlighted the essential role of GNDF, bFGF, and LIF for the differentiation of PGCLCs to SSCLCs, and the requirement of RA for its further differentiation into SLCs. Nevertheless, we should note here that the induction efficiency in our system is also not very high, which would be improved in further studies.

Eif2s $3 y$, which encodes the gamma-subunit of the eukaryotic translation initiation factor EIF2, is essential for normal spermatogonial proliferation and progression through meiotic prophase [15]. Previous studies have demonstrated that live offspring can be obtained using germ cells from male mice with the Y chromosome contribution limited to only Sry and Eif $2 s 3 y$, and indicated that Eif $2 s 3 y$ was the only gene necessary for driving spermatogenesis through the first meiotic division [9]. In our previous study, we found the novel role of Eif2s3y in the regulation of ESC pluripotency maintenance and proliferation control, and we also supported its role in meiosis, in accordance with findings of some other results [5, 8, 27]. Therefore, we take the advantage of Eif $2 s 3 y$ in our induction system. We found that Eif2s3y was of critical importance in the differentiation of PGCLCs into SSCLCs and further into SLCs. Thus, our results further highlighted the essential role of Eif $2 s 3 y$ in enhancing meiosis derived from ESCs.

Taken together, we have demonstrated an efficient approach toward the stepwise differentiation of ESCs into haploid SLCs in vitro and this process was significantly enhanced by Eif2s3y. For the first time, our study provided detailed evidences for the differentiation of PGCLSCs into SSCLCs and SLCs in vitro with all defined factors. This work would potentially contribute to investigations concerning the generation of haploid human spermatids in vitro as well as gene therapies for male infertility. 


\section{Materials and methods}

\section{Male germline cell induction system}

The basal culture medium is consisted of Neurobasal ${ }^{\mathrm{TM}}$ (Gibco, Waltham, Massachusetts, USA) and DMF/12 medium (Hyclone, Logan, Utah, USA) (1:1), supplemented with N-2 Supplement (100x) (Gibco) and B-27 ${ }^{\mathrm{TM}}$ Supplement (50x) (Gibco). The induction of ESCs into EpiLCs and PGCLCs was modified from a previously published protocol [1]. For EpiLC induction, $1 \times 10^{5}$ ESCs were plated on wells of human plasma fibronectin-coated 12-well-plates in basal culture medium containing Activin A (20 ng/ml; PeproTech, Rocky Hill, NJ, USA), bFGF (12 ng/ml; PeproTech), and KSR (1\%; Gibco). For PGCLC differentiation, $1 \times 10^{5}$ day 2 induced cells were reseeded on mouse embryonic fibroblast (MEF) feeder layer $\left(1 \times 10^{5}\right.$ cells per well of a 12-well-plate) in basal culture medium containing BMP4 $(50 \mathrm{ng} / \mathrm{ml}$; PeproTech), SCF (50 ng/ml; Sino Biological, Beijing, China), Bmp8a (50 ng/ml, R\&D Systems, Abingdon, UK), insulin (12 $\mu \mathrm{g} / \mathrm{ml}$; Sigma-Aldrich, St. Louis, Missouri, United States) and $10 \%$ KSR for 3 days. For SSCLC differentiation, $3 \times 10^{5}$ day 5 -induced cells were reseeded on an feeder layer $\left(3 \times 10^{5}\right.$ cells per well of a 6-well-plate) in basal culture medium containing BMP4 (25 ng/ml; PeproTech), bFGF (20 ng/ml; PeproTech), GDNF (40 ng/ml, PeproTech), LIF (10 ng/ml; Miltenyi Biotec, Bergisch Gladbach, Germany), insulin (12 $\mu \mathrm{g} / \mathrm{ml}$; Sigma-Aldrich) and 15\% KSR for 6 days. Then, $5 \times$ $10^{5}$ day 11-induced cells were reseeded on an feeder layer in a 6-well-plate in basal culture medium containing $2 \mathrm{nM}$ RA (Sigma-Aldrich) and 15\% KSR for 12 days.

\section{Real-time PCR and analysis}

RNA was extracted with TRIzol (Takara, Kusatsu, Japan) as previously reported [28]. Reverse transcription was performed using a reverse transcription kit (Thermo Fisher Scientific, Waltham, Massachusetts, USA). The RT-PCR primers used in this article are listed in Table S2.

\section{Immunofluorescence staining}

Immunofluorescence staining was conducted as previously reported [5]. The antibodies used in this article are listed as follows: anti-SSEA-1 (1:500; Bioss, Woburn, MA, USA), anti-Prdm1 (1:50; Bioss), anti-Prdm14 (1:300; SigmaAldrich), anti-AP2 $\gamma$ (1:200; Bioss), anti-PLZF (1:400; Abcam, Cambridge, UK), anti-GFR $\alpha$ 1(1:200; Abcam), anti$\operatorname{VASA}(1: 200$; Abcam), anti-c-Kit (1:500; Boster, Wuhan, China), anti-SYCP3 (1:400; Abcam), fluorescein isothiocyanate (FITC)-conjugated secondary antibody (1:500; Chemi-Con, Billerica, Massachusetts, USA), and Hoechst 33342 (Sigma-Aldrich).

\section{Western blot}

The western blot protocol was the same as that in a previous article [5]. Detailed information of the antibodies is as follows: anti-Acrosin (1:500; Abcam), anti-PRM1 (1:400; Santa Cruz, Dallas, TX, USA), anti-SYCP3 (1:400; Abcam), anti-GAPDH (1:5000; Genesci, Beijing, China), horse-radish peroxidase-conjugated anti-rabbit antibody (1:3000; Boster), and anti-mouse antibody (1:2000; Boster).

\section{Flow cytometry}

For haploid analysis, a commercially available kit (Cell Cycle Staining Kit, LiankeBio, Hangzhou, China) was used according to the manufacturer's instructions. For staining of CD61 and SSEA-1, cells were incubated with anti-CD16/32 (Biolegend, San Diego, CA, USA) for $20 \mathrm{~min}$, and subsequently treated for $15 \mathrm{~min}$ with FITC-conjugated CD61 antibody (Biolegend) and Alexa Fluor 647-conjugated SSEA-1 antibody (BD Biosciences, Franklin Lakes, New Jersey, USA). For intracellular VASA and PLZF staining, cells were treated with a Foxp3/Transcription Factor Staining Buffer Set (eBioscience, San Diego, CA, USA) according to the manufacturer's instructions. Anti-VASA antibody was purchased from Abcam, and Alexa Fluor 647conjugated secondary antibody was purchased from Life Technologies (Waltham, MA, USA). PE-conjugated PLZF antibody was purchased from eBioscience. Flow cytometry was conducted with a FACSVerse flow cytometer (BD Biosciences), and the results were analyzed using a FlowJo software (Tree Star, Inc., Ashland, OR, USA).

\section{Cell transplantation}

The mice used in this article were ICR mice purchased from the animal center of the Fourth Military Medical University in Xi' an. Seven-week-old male ICR mice were treated with busulfan at dose of $30 \mathrm{mg} / \mathrm{kg}$ for 3 weeks to be rendered infertile. The cell transplantation protocol was conducted as previously reported [5]. In brief, one mouse testis was injected with $3 \times 10^{5}$ cells in $15 \mu \mathrm{l}$ basal culture medium (day 11-induced cells from wild-type ESCs in Fig. 3; day 11-induced cells from Vector- or Eif2s3y-overexpressing ESCs in Fig. 5 and uninduced wild-type ESCs in Fig. S2); the other one was injected with only $15 \mu \mathrm{l}$ basal culture medium. After 2 months, some mice were sacrificed for testis harvest, and the organs were fixed in $4 \%$ formaldehyde overnight for further analysis; other mice were mated with female mice to produce offspring. The primers used for offspring detection are listed in Table S1.

All mouse experiments were conducted according to the NWSUAF guideline of Animal Care and Use Committee. 


\section{Statistical analysis}

All results were replicated at least three times. The data presented herein are the means $\pm \mathrm{SD}$. To compare significant differences, a two-tailed Student's $t$ test was used, and the difference was significant if the $p$ value was less than $0.05(* p<0.05, * * p<0.01, * * * p<0.001)$.

Acknowledgments We appreciate Dr. Christian Dani of University of Nice Sophia Antipolis for excellent comments and revisions. This work was supported by grants from the China National Basic Research Program (JFYS 2016YFA0100203), Doctoral Scientific Research Foundation of 2017 (Z109021715), the Program of National Natural Science Foundation of China (31572399 and 31272518) and China Postdoctoral Science Foundation (K3080217064).

Author contributions N.L., W.T.M., Q.Y.S., M.F.Z., and W.Q.L. performed the experiments. N.L., W.T.M., Z.Y.D., C.Y.W., and B.W.N. analyzed the data. J.L.H. designed the project. N.L. and W.T.M. wrote the manuscript, and J.L.H. revised the manuscript.

\section{Compliance with ethical standards}

Conflict of interest The authors declare that they have no conflict of interest.

Publisher's note: Springer Nature remains neutral with regard to jurisdictional claims in published maps and institutional affiliations.

\section{References}

1. Hayashi K, Ohta H, Kurimoto K, Aramaki S, Saitou M. Reconstitution of the mouse germ cell specification pathway in culture by pluripotent stem cells. Cell. 2011;146:519-32.

2. Ishikura $Y$, Yabuta $Y$, Ohta H, Hayashi K, Nakamura T, Okamoto I, et al. In vitro derivation and propagation of spermatogonial stem cell activity from mouse pluripotent stem cells. Cell Rep. 2016;17:2789-804.

3. Zhou Q, Wang M, Yuan Y, Wang X, Fu R, Wan H, et al. Complete meiosis from embryonic stem cell-derived germ cells in vitro. Cell Stem Cell. 2016;18:330-40.

4. Zhao Y, Ye S, Liang D, Wang P, Fu J, Ma Q, et al. In Vitro modeling of human germ cell development using pluripotent stem cells. Stem Cell Rep. 2018;10:509-23.

5. Li N, Mu H, Zheng L, Li B, Wu C, Niu B, et al. EIF2S3Y suppresses the pluripotency state and promotes the proliferation of mouse embryonic stem cells. Oncotarget. 2016;7: 11321-31.

6. Yamauchi Y, Riel JM, Ruthig VA, Ortega EA, Mitchell MJ, Ward MA. Two genes substitute for the mouse Y chromosome for spermatogenesis and reproduction. Science. 2016; 351:514-6.

7. Murata C, Sawaya H, Nakata K, Yamada F, Imoto I, Kuroiwa A. The cryptic Y-autosome translocation in the small Indian mongoose, Herpestes auropunctatus, revealed by molecular cytogenetic approaches. Chromosoma. 2016;125:807-15.

8. Yamauchi Y, Riel JM, Ruthig V, Ward MA. Mouse Y-encoded transcription factor Zfy2 is essential for sperm formation and function in assisted fertilization. PLoS Genet. 2015;11: e1005476.
9. Yamauchi Y, Riel JM, Stoytcheva Z, Ward MA. Two Y genes can replace the entire $\mathrm{Y}$ chromosome for assisted reproduction in the mouse. Science. 2014;343:69-72.

10. Goodyear S, Brinster R. Spermatogonial stem cell transplantation to the testis. Cold Spring Harb Protoc. 2017;2017:pdbprot094235.

11. Ganguli N, Wadhwa N, Usmani A, Kunj N, Ganguli N, Sarkar RK, et al. An efficient method for generating a germ cell depleted animal model for studies related to spermatogonial stem cell transplantation. Stem Cell Res Ther. 2016;7:142.

12. Kanatsu-Shinohara M, Ogonuki N, Inoue K, Miki H, Ogura A, Toyokuni S, et al. Long-term proliferation in culture and germline transmission of mouse male germline stem cells. Biol Reprod. 2003;69:612-6.

13. Griswold MD, Hogarth CA, Bowles J, Koopman P. Initiating meiosis: the case for retinoic acid. Biol Reprod. 2012;86:35.

14. Jan SZ, Hamer G, Repping S, de Rooij DG, van Pelt AM, Vormer TL. Molecular control of rodent spermatogenesis. Biochim Biophys Acta. 2012;1822:1838-50.

15. Mazeyrat S, Saut N, Grigoriev V, Mahadevaiah SK, Ojarikre OA, Rattigan A, et al. A Y-encoded subunit of the translation initiation factor Eif 2 is essential for mouse spermatogenesis. Nat Genet. 2001;29:49-53.

16. Geijsen N, Horoschak M, Kim K, Gribnau J, Eggan K, Daley GQ. Derivation of embryonic germ cells and male gametes from embryonic stem cells. Nature. 2004;427:148-54.

17. Saitou M, Miyauchi H. Gametogenesis from pluripotent stem cells. Cell Stem Cell. 2016;18:721-35.

18. Eguizabal C, Montserrat N, Vassena R, Barragan M, Garreta E, Garcia-Quevedo L, et al. Complete meiosis from human induced pluripotent stem cells. Stem Cells. 2011;29:1186-95.

19. Irie N, Weinberger L, Tang WWC, Kobayashi T, Viukov S, Manor YS, et al. SOX17 is a critical specifier of human primordial germ cell fate. Cell. 2015;160:253-68.

20. Magnusdottir E, Dietmann S, Murakami K, Gunesdogan U, Tang F, Bao S, et al. A tripartite transcription factor network regulates primordial germ cell specification in mice. Nat Cell Biol. 2013;15:905-15.

21. Nakaki F, Hayashi K, Ohta H, Kurimoto K, Yabuta Y, Saitou M. Induction of mouse germ-cell fate by transcription factors in vitro. Nature. 2013;501:222-6.

22. Yokonishi T, Sato T, Katagiri K, Komeya M, Kubota Y, Ogawa T. In vitro reconstruction of mouse seminiferous tubules supporting germ cell differentiation. Biol Reprod. 2013;89:15.

23. Kubota H, Avarbock MR, Brinster RL. Growth factors essential for self-renewal and expansion of mouse spermatogonial stem cells. Proc Natl Acad Sci USA. 2004;101:16489-94.

24. Wang S, Wang X, Ma L, Lin X, Zhang D, Li Z, et al. Retinoic acid is sufficient for the in vitro induction of mouse spermatocytes. Stem Cell Rep. 2016;7:80-94.

25. Yang S, Yuan Q, Niu M, Hou J, Zhu Z, Sun M, et al. BMP4 promotes mouse iPS cell differentiation to male germ cells via Smad1/5, Gata4, Id1 and Id2. Reproduction. 2017;153:211-20.

26. Endo T, Romer KA, Anderson EL, Baltus AE, de Rooij DG, Page DC. Periodic retinoic acid-STRA8 signaling intersects with periodic germ-cell competencies to regulate spermatogenesis. Proc Natl Acad Sci USA. 2015;112:E2347-56.

27. Matsubara Y, Kato T, Kashimada K, Tanaka H, Zhi Z, Ichinose S, et al. TALEN-mediated gene disruption on $\mathrm{Y}$ chromosome reveals critical role of EIF2S3Y in mouse spermatogenesis. Stem Cells Dev. 2015;24:1164-70.

28. Li M, Liu C, Zhu H, Sun J, Yu M, Niu Z, et al. Expression pattern of Boule in dairy goat testis and its function in promoting the meiosis in male germline stem cells (mGSCs). J Cell Biochem. 2013;114:294-302. 